\title{
Breached pairing in trapped three-color atomic Fermi gases
}

\author{
Beatriz Errea, ${ }^{1}$ Jorge Dukelsky, ${ }^{1}$ and Gerardo Ortiz ${ }^{2}$ \\ ${ }^{1}$ Instituto de Estructura de la Materia-CSIC, Serrano 123, 28006 Madrid, Spain \\ ${ }^{2}$ Department of Physics, Indiana University, Bloomington, Indiana 47405, USA
}

(Received 11 December 2008; revised manuscript received 10 February 2009; published 13 May 2009)

\begin{abstract}
We introduce an exactly solvable model for trapped three-color atom gases. Application to a system of cigar-shaped trapped cold fermions reveals a complex structure of breached pairing phases. We find two competing superfluid phases at weak and intermediate couplings, each with two-color pair condensates that can be distinguished with density profile measurements.
\end{abstract}

DOI: 10.1103/PhysRevA.79.051603

PACS number(s): 03.75.Ss, 03.75.Mn, 05.30.Fk, 02.30.Ik

Color superconductivity is predicted to occur in quark matter at sufficiently high density and low temperatures [1]. Quarks, having three different colors [red $(R)$, green $(G)$, and blue $(B)]$ and a strong attractive interaction, allow for more diverse pairing patterns compared to the $\mathrm{SU}(2)$ Cooper pairing in classical metallic superconductors. Such diversity, likewise, makes it hard to establish the particular pairing symmetry favored by nature. With the advent of ultracold trapped Fermi gases a window of opportunities has opened to address some of these fundamental questions, at least, in a qualitative fashion (see, for example, [2]). One can certainly manipulate different atomic species and hyperfine states to effectively generate multicolor Fermi gases with attractive interactions.

The goal of this Rapid Communication is to investigate the superfluid behavior of an imbalanced three-color Fermi gas by means of an exactly solvable pairing model. We will present an exactly solvable color-pairing Hamiltonian derived from the $\mathrm{SO}(6)$ quadratic invariants of the generalized Richardson-Gaudin (RG) models $[3,4]$. Previous studies using standard mean-field [5], density-matrix renormalization-group [6], or Bethe ansatz [7] technique concentrated on the competition between a trionic or baryonic phase and a color superfluid phase. A main result of our work is the competition between breached-pair (BP) and unbreached-pair (UP) superfluid phases in a polarized multicolor Fermi gas. As in the two-color case, where density profiles have been recently investigated experimentally [8] and theoretically [9], an analog of the BP or Sarma phase $[10,11]$ appears. We find a complex structure of breached pairing as well as the coexistence of two pair condensates. While the possibility of coexistence of several superfluid phases has been suggested in [12] using a localdensity approximation (LDA) theory, we predict the existence of two distinct color fermionic condensates and propose ways to detect them. Within our model this is a genuine effect, although care must be exercised when contrasted to experiments since interactions not included in our model could make this phase unstable against the formation of a fraction of bound trions in the strong-coupling limit. However, population imbalance as well as the experimental realization of a stable three-color atomic gas with different atomic masses and/or different Feshbach resonances $[13,14]$ could stabilize it.

Consider the SU(3) color-symmetric Hamiltonian

$$
H=\sum_{i}^{L} \varepsilon_{i} N_{i}-g \sum_{i i^{\prime}}^{L} \sum_{\alpha} A_{i \alpha}^{\dagger} A_{i^{\prime} \alpha}
$$

for $L$ levels $i$ of energy $\varepsilon_{i}$, where $\alpha=(R, G, B)$ is the color index, $N_{i}=\Sigma_{\alpha} N_{i \alpha}$ is the number operator of the orbit $i, A_{i \alpha}^{\dagger}=\Sigma_{\beta \gamma} \varepsilon_{\alpha \beta \gamma} a_{i \beta}^{\dagger} a_{i \gamma}^{\dagger}, A_{i \alpha}=\left(A_{i \alpha}^{\dagger}\right)^{\dagger}$, are the pair creators, and $g>0$ is the pairing strength. Here $a_{i \beta}^{\dagger}$ creates a (canonical) fermionic atom in level $i$ with color $\beta$ and $\varepsilon_{\alpha \beta \gamma}$ is the completely antisymmetric tensor in color space. The $\mathrm{SO}(6)$ algebra (15 generators) is completed by the nine particle-hole operators $C_{i, \alpha \beta} \equiv a_{i \alpha}^{\dagger} a_{i \beta}$. These nine operators, which include the number operators for the three different colors in the level $i, N_{i \alpha}=C_{i, \alpha \alpha}$, close an $\mathrm{U}(3)$ subalgebra of $\mathrm{SO}(6)$.

Hamiltonian (1) has equal pairing strengths $g$ (or scattering lengths) and equal single-particle energies (masses) for the three colors. It describes a three-color Fermi gas with attractive contact interactions in the low-density limit, truncated to include only the scattering of zero-momentum pairs. As such, it is the natural generalization of the BCS Hamiltonian extensively used to describe the BCS-Bose-Einstein condensate (BEC) crossover in two-color atomic gases. Moreover, the SU(3) symmetry is preserved and, thus, the eigenstates are organized in degenerate $\mathrm{SU}(3)$ multiplets. The SU(3) symmetry, however, may be broken by choosing a different combination of integrals of motion and, for example, one can generate an integrable model of atoms with unequal masses [15].

The exact solution of the SO(6) RG model depends on three sets of spectral parameters since the algebra is of rank 3 . The first set includes the usual pair energies $e_{\alpha}$ of the $\mathrm{SO}(6)$ algebra, while the other two, composed of the spectral parameters $\omega_{\alpha}$ and $\gamma_{\alpha}$, are associated with the $\mathrm{SU}(3)$ subalgebra of $\mathrm{SO}(6)$. They satisfy the generalized Richardson equations, 


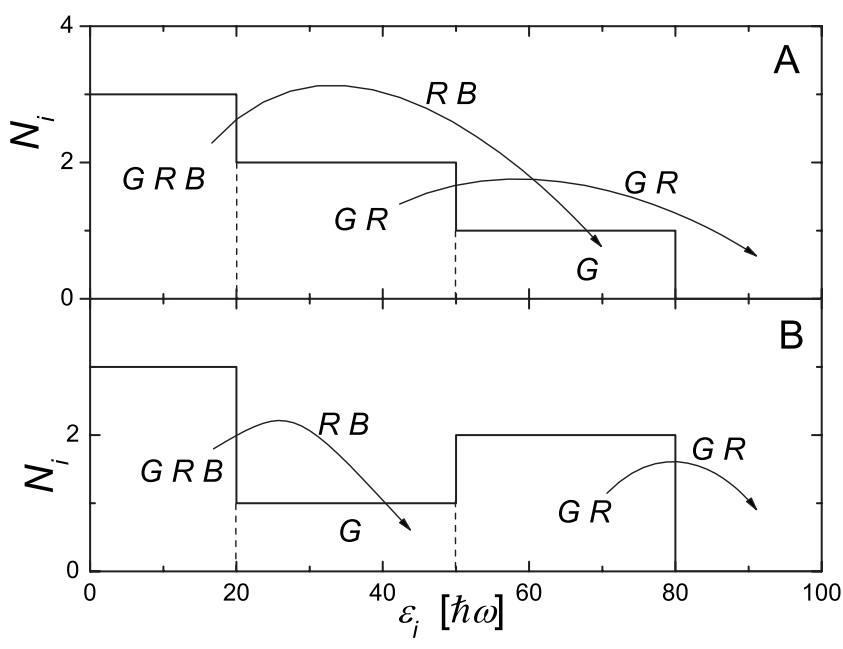

FIG. 1. Occupation numbers for (A) the BP state and (B) the UP state, showing the allowed pair scatterings.

$$
\begin{gathered}
\sum_{\beta(\neq \alpha)}^{M} \frac{2}{e_{\beta}-e_{\alpha}}-\sum_{\beta}^{N_{B}} \frac{1}{\omega_{\beta}-e_{\alpha}}+\sum_{i}^{L} \frac{\nu_{i}-1}{2 \varepsilon_{i}-e_{\alpha}}=-\frac{1}{4 g}, \\
\sum_{\beta(\neq \alpha)}^{N_{B}} \frac{2}{\omega_{\beta}-\omega_{\alpha}}-\sum_{\beta}^{M} \frac{1}{e_{\beta}-\omega_{\alpha}}-\sum_{\beta}^{Q} \frac{1}{\gamma_{\beta}-\omega_{\alpha}}=0, \\
\sum_{\beta(\neq \alpha)}^{Q} \frac{2}{\gamma_{\beta}-\gamma_{\alpha}}-\sum_{\beta}^{N_{B}} \frac{1}{\omega_{\beta}-\gamma_{\alpha}}-\sum_{i}^{L} \frac{\nu_{i}}{2 \varepsilon_{i}-\gamma_{\alpha}}=0 .
\end{gathered}
$$

The number of spectral parameters in each set is determined by the number of particles of each color $N_{\alpha}$ and by the total seniority quantum number $\nu: M=(N-\nu) / 2$ and $Q=\left(N_{B}+N_{R}-N_{G}+\nu\right) / 2$, where we have assumed, without loss of generality, $N_{G} \geq N_{R} \geq N_{B}$. The seniority of level $i, \nu_{i}$, counts the number of unpaired fermions and is defined from $A_{i \alpha}\left|\nu_{i}\right\rangle=0$ and $N_{i}\left|\nu_{i}\right\rangle=\nu_{i}\left|\nu_{i}\right\rangle$, where $\nu_{i}=0,1$. The total seniority is $\nu=\Sigma_{i} \nu_{i}$. Hamiltonian (1) preserves the seniority since it can create or destroy pairs of particles conserving the number parity of the level, i.e., for a given configuration each level has an even (odd) number of particles where $\nu_{i}=0$ (1).

The eigenvalues of Hamiltonian (1) are $E=\sum_{\alpha}^{M} e_{\alpha}$ $+\sum_{i=1}^{L} \varepsilon_{i} \nu_{i}$. The corresponding eigenfunctions, although, are determined by the three sets of parameters and define a complete Hilbert-space basis of the system.

Consider a gas composed of $N=N_{G}+N_{R}+N_{B}$ fermionic atoms. In the weak-coupling limit the energy levels are filled up to the Fermi energy for each color $\varepsilon_{N_{\alpha}}$, a situation depicted in Fig. 1(A) for $N_{G}=80, N_{R}=50$, and $N_{B}=20$. In this case the seniorities $\nu_{i}$ are equal to 1 for $i \leq 20$ and $50<i \leq 80$ and they are 0 for $20<i \leq 50$ and $i>80$, defining a clear separation of the Hilbert space into regions of odd particle states $\left(\nu_{i}=1\right)$ and regions of even particle states $\left(\nu_{i}=0\right)$. When the pairing interaction $g$ is switched on, $R-B$ pairs from the first region $(i \leq 20)$ cannot scatter to the second region $(20<i \leq 50)$ due to Pauli blocking and they have to jump this forbidden region to scatter into the third region $50<i \leq 80$. Analogously, $G-R$ pairs of the second region

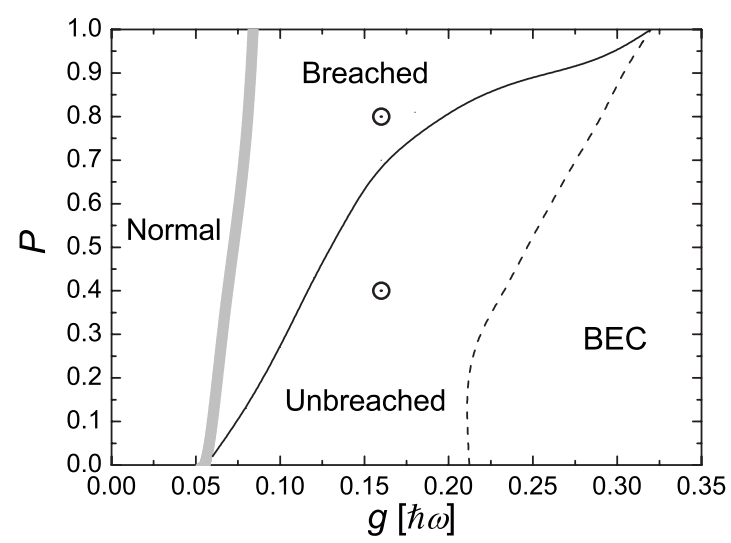

FIG. 2. Phase diagram of a 1D partially polarized trapped Fermi gas with $N=150, N_{R}=50, P=\left(N_{G}-N_{B}\right) /\left(N_{G}+N_{B}\right)$, and $L=500$. The dashed line signals the crossover to a mixed color BEC region.

have to jump the third forbidden region to scatter into the fourth region $(i>80)$. This configuration, that we call $\mathrm{BP}$ state, turns out to be the ground state (GS) at weak coupling. For larger values of $g$ other configurations compete with the $\mathrm{BP}$ state. Those configurations, at the cost of increasing their kinetic energy, reduce the effect of Pauli blocking, therefore facilitating the pair scattering into interior level regions. In particular, the UP state depicted in panel (B) has no blocked interior region and will be the GS of the system at strong coupling. Therefore, the physical mechanism determining the superfluid phase boundaries is the competition between the kinetic and pairing energies of the fermionic atoms. Note that there are many variants of BP and UP states. Each of them is determined by a given initial noninteracting configuration, i.e., that is by a set of seniorities $\nu_{i}$. We checked numerically that the considered BP and the UP states are the GS at weak and strong pairing strengths, respectively, and the other possible configurations constitute excited states for all values of the interaction strength.

Although Hamiltonian (1) is exactly solvable in any dimension, for simplicity we will consider a system of $N=150 \quad\left(N_{R}=50\right)$ fermionic atoms trapped by a onedimensional (1D) harmonic potential (of frequency $\omega$ ) with an energy cutoff at $E_{\text {cut }}=500 \hbar \omega$, implying $L=500$ threefolddegenerate single-particle levels. The Richardson equations (2) can be solved numerically starting with an analytical solution in the week-coupling limit and iteratively increasing the constant $g$, using the solution of the previous step as the new initial guess, up to the desired value of $g$. We performed extensive calculations to determine the quantum phase diagram of this system as a function of the pairing strength $g$ and the polarization $P=\left(N_{G}-N_{B}\right) /\left(N_{G}+N_{B}\right)$.

Two-color superfluid phases emerge as a function of color asymmetry and pairing strength (see Fig. 2). A first-order quantum phase transition, due to level crossing, separates the BP and UP superfluid phases, which are labeled by different sets of seniority quantum numbers. On the other hand, there is a smooth crossover between the two superfluid phases and a normal fluctuation-dominated state depicted by a thick gray line [16]. The normal Fermi-liquid-type state is dominated by pairing fluctuations which are fully taken into account by the exact solution. We adopted the criterion that the normal 


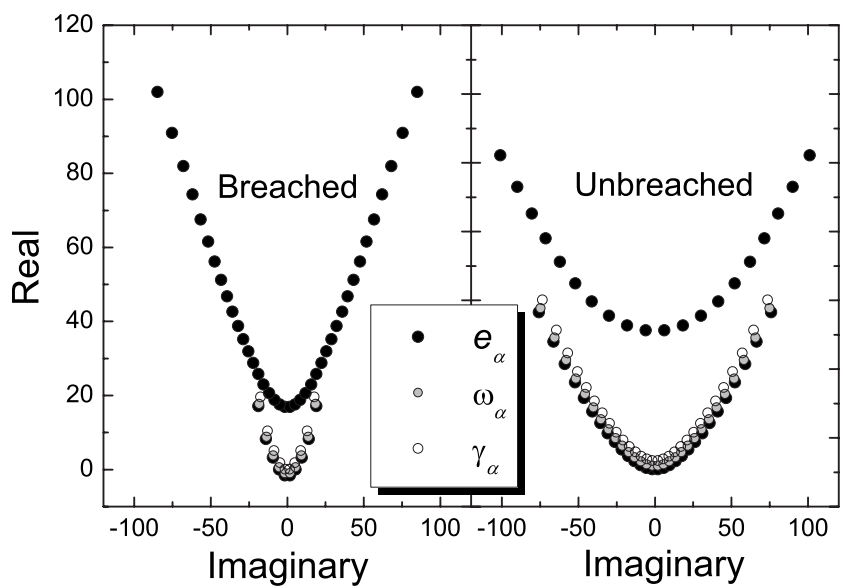

FIG. 3. Spectral parameters (in units of $\hbar \omega$ ) of the BP state with $P=0.8$ and the UP state with $P=0.4$, for $g=0.16$.

region extends, for a given $P$, from $g=0$ up to the value of $g$ for which $10 \%$ of the pair energies $e_{\alpha}$ are complex, meaning that the condensate fraction $f$ is $\leq 0.1$ [17]. The BEC region (see Fig. 2) is characterized by spectral parameters having negative real parts, i.e., all pairs represent quasibound molecules of mixed color as opposed to the BCS region with condensate pairs of definite color (see below). To study the correlations and structure of the BP and the UP states we have chosen two particular points, marked with open circles in Fig. 2. Within our model seniority is a conserved quantum number, disfavoring the formation of bound trionic molecular states which are more likely to appear for very low $P$ and stronger coupling.

Figure 3 shows the three sets of spectral parameters for these two states. Note that for this value of $g=0.16$ all pair energies are complex with a positive real part, implying that the condensate fraction is $f=1$, and all pairs behave as Cooper resonances as opposed to bound molecules of a BEC [17]. In both cases the pair energies form two separate arcs in the complex plane, indicating the existence of two-color fermionic pair condensates. In the strong-coupling limit the two arcs coalesce into a single arc corresponding to a single BEC condensate with negative real part of their pair energies. The lower arc is overlapping with arcs of the two other spectral parameters $\omega_{\alpha}$ and $\gamma_{\alpha}$ which account for the couplings in the $\mathrm{SU}(3)$ color subspace. The interpretation is that the upper arc of isolated pair energies $e_{\alpha}$ describes $G-R$ Cooper pairs, while the lower arc corresponds to $R-B$ Cooper pairs. An analysis in terms of the eigenvalues of the two-body density matrix would lead to a macroscopic eigenvalue of the $G-R$ and the $R-B$ pair density matrices and no macroscopic eigenvalue in the $G-B$ pair density matrix. The appearance of these two condensates will be reflected in the occupation probabilities that can be calculated using the HellmannFeynman theorem on the integrals of motion as will be explained in a forthcoming paper [18].

The occupation probabilities, $\left\langle N_{i}\right\rangle$, for both states are depicted in Fig. 4. In the BP state panel we see how the $G-R$ pairs avoid the region $50<i \leq 90$, Pauli blocked by the $G$ atoms, to scatter off into the region $i>90$. Analogously, although less evident due to the smaller number of pairs, the

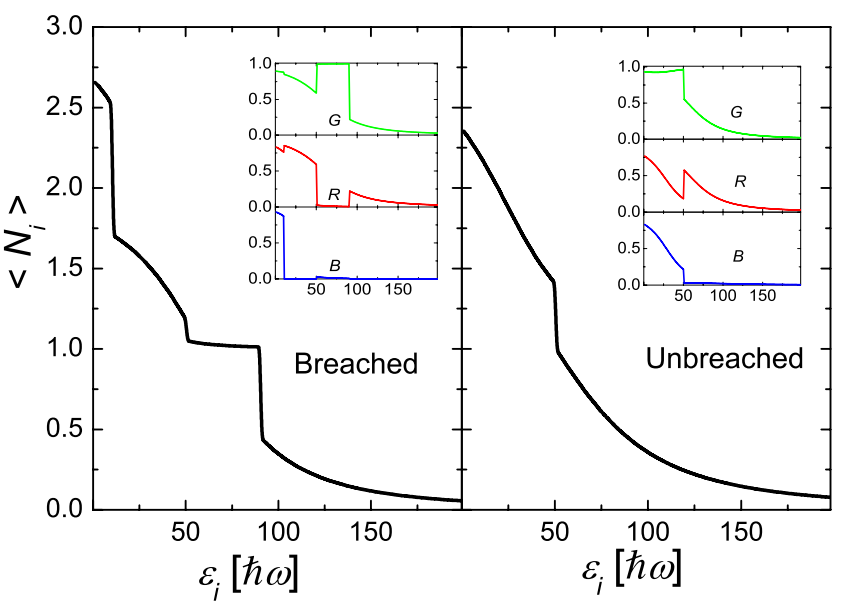

FIG. 4. (Color online) Occupation numbers of the BP state with $P=0.8$ and the UP state with $P=0.4$, for $g=0.16$. In the inset we display the different color contributions to $\left\langle N_{i}\right\rangle$.

ten $R-B$ pairs avoid the region $10<i \leq 50$, blocked by the $R$ atoms, to scatter off into the region $i>50$. We may realize that in the latter case the blocking is not perfect, unlike in the two-component gases, because of the depletion of the $R$ atoms in the $10<i \leq 50$ region due to $G-R$ pairing. The $G-B$ pairing is prevented from being realized due to two consecutive blocked regions. On the contrary, in the UP state pairs do not have to avoid blocked regions. The $G-R$ pairs in the $50<i \leq 70$ region scatter off into the regions $i>70$, and $R-B$ pairs in the region $i \leq 30$ scatter off into the region $30<i \leq 50$ as well as into the depleted region $i>50$. These two different physical scenarios manifest in the arc geometry of the spectral parameters in Fig. 3. While the BP state has an inner condensate of ten $R-B$ and an outer condensate of 40 $G-R$ pairs, the UP state has 30 condensed $R-B$ and 20 condensed $G-R$ pairs.

An experimental way to uncover the nature of the color superfluid correlations consists in measuring the density

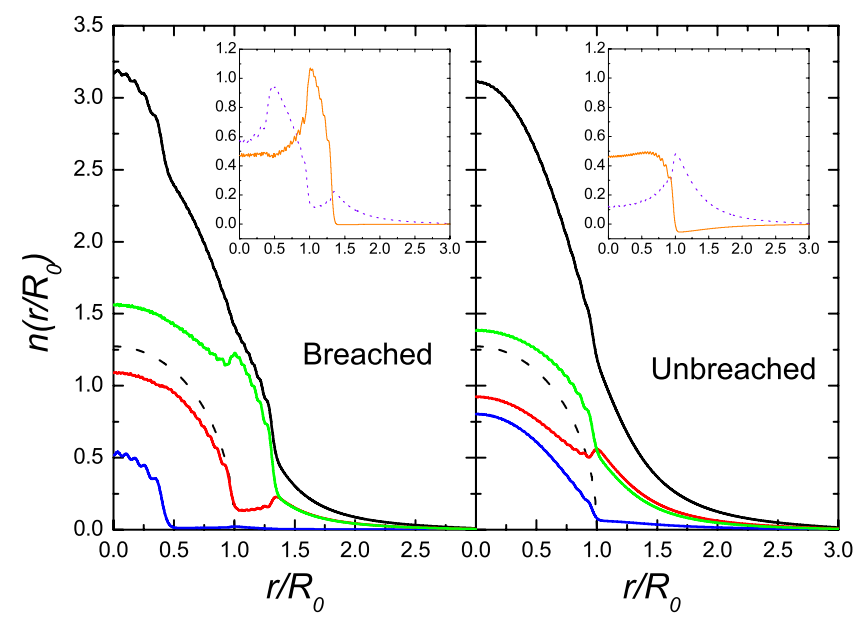

FIG. 5. (Color online) Radial density profiles for the BP and UP states for $g=0.16$. The dashed curve corresponds to the ThomasFermi approximation for the $R$ atoms. The insets show the density differences between the $R$ and $B$ (dashed dark gray curve) and $G$ and $R$ (solid light gray curve) species. 
clouds of the trapped Fermi gas. Figure 5 shows the radial density profiles for these two states normalized to the $R$ density as a function of the distance $r$ from the center of the trap, in units of the Thomas-Fermi radius $R_{0}$ of the $R$ species. The insets display the density differences between the $R$ and $B$ (dashed dark gray curve) and $G$ and $R$ (solid light gray curve) species. In both cases the outer region is dominated by $G-R$ pairing that enforces a maximum overlap of the $G$ and $R$ components of the wave function. However, the BP state shows a richer structure in the inner region. In particular, a clear signal of a BP state appears as a peak in the density profile of the majority atoms $G$, which is magnified in the $G$ and $R$ density difference. Two peaks appear in the $R$ and $B$ density difference consistent with the decay and revival of the $B$ density in regions of $R-B$ pairing. The structure of the density differences is smoother in the UP phase due to the absence of breached pairing. Regions of constant energy differences signal the dominance of the corresponding pairing phase. In the UP state for $0.0<r / R_{0}<0.5$ it is dominated by a $R-B$ superfluid, while for $r / R_{0}>1.0$ we have a $G-R$ superfluid.
One might measure each of the two-color pair condensate's fractions by exploiting the differences in the colorpair-dependent Feshbach resonances [14]. It would then be possible to use the ramp technique as described in [19], sweeping the magnetic field such that only one class of pairs are transformed into bound molecules, allowing for the determination of the corresponding fraction of the condensate. A bigger ramp would then transform all pairs into molecules, therefore allowing measurement of the complete fraction of the condensate.

Addition of an optical lattice potential could add new exotic phases such as the crystallization of trions [20] or color Fulde-Ferrell-Larkin-Ovchinnikov phases. The latter could be incorporated to this exactly solvable model as a generalization of [21], but this is beyond the scope of this Rapid Communication.

We acknowledge fruitful discussions with G. G. Dussel. This work was supported in part by Grant No. FIS200612783-C03-01 of the Spanish DGI. B.E. was supported by the Spanish CE-CAM.
[1] M. G. Alford, A. Schmitt, K. Rajagopal, and T. Schäfer, Rev. Mod. Phys. 80, 1455 (2008).

[2] S. Giorgini, L. P. Pitaevskii, and S. Stringari, Rev. Mod. Phys. 80, 1215 (2008).

[3] A. G. Ushveridze, Quasi-Exactly Solvable Models in Quantum Mechanics (IOP, Bath, 1994).

[4] M. Asorey, F. Falceto, and G. Sierra, Nucl. Phys. B 622, 593 (2002).

[5] A. Rapp, G. Zarand, C. Honerkamp, and W. Hofstetter, Phys. Rev. Lett. 98, 160405 (2007); T. Paananen, J.-P. Martikainen, and P. Törmä, Phys. Rev. A 73, 053606 (2006).

[6] S. Capponi, G. Roux, P. Lecheminant, P. Azaria, E. Boulat, and S. R. White, Phys. Rev. A 77, 013624 (2008).

[7] X. W. Guan, M. T. Batchelor, C. Lee, and H.-Q. Zhou, Phys. Rev. Lett. 100, 200401 (2008); X.-J. Liu, H. Hu, and P. D. Drummond, Phys. Rev. A 77, 013622 (2008).

[8] Y. Shin, M. W. Zwierlein, C. H. Schunck, A. Schirotzek, and W. Ketterle, Phys. Rev. Lett. 97, 030401 (2006).

[9] G. Bertaina and S. Giorgini, Phys. Rev. A 79, 013616 (2009).

[10] N. V. Sarma, Phys. Lett. A 25, 315 (1967).

[11] W. V. Liu and F. Wilczek, Phys. Rev. Lett. 90, 047002 (2003).

[12] T. Paananen, P. Törmä, and J.-P. Martikainen, Phys. Rev. A 75, 023622 (2007).
[13] T. B. Ottenstein et al., Phys. Rev. Lett. 101, 203202 (2008).

[14] J. H. Huckans et al., Phys. Rev. Lett. 102, 165302 (2009.

[15] The case of atomic species with different masses can also be exactly solved within the Gaudin algebra. Since this is of experimental relevance we write down the Hamiltonian, $\quad H=\sum_{i}^{L} \varepsilon_{i}\left[N_{i G}+N_{i B}+(1+\Delta) N_{i R}\right]-g \sum_{i i^{\prime}}^{L} \Sigma_{\alpha} A_{i \alpha}^{\dagger} A_{i^{\prime} \alpha}$ $-2 g \sum_{i i^{\prime}}^{L}, \Sigma_{\alpha \neq \beta} C_{i \alpha \beta} C_{i^{\prime} \beta \alpha}$, where $\Delta$ quantifies the difference in the atomic masses.

[16] A quantum phase diagram is usually defined in the thermodynamic limit. In that limit the transition to a superfluid state at $P=0$ takes place at $g=0$ and, therefore, it is expected that the boundary between the normal and the superfluid phases will move to the left defining the Chandrasekhar-Clogston limit of a three-color Fermi gas.

[17] G. Ortiz and J. Dukelsky, Phys. Rev. A 72, 043611 (2005).

[18] B. Errea, et al. (unpublished).

[19] C. A. Regal, M. Greiner, and D. S. Jin, Phys. Rev. Lett. 92, 040403 (2004); M. W. Zwierlein et al., ibid. 92, 120403 (2004).

[20] R. A. Molina, J. Dukelsky, and P. Schmitteckert, e-print arXiv:0807.1886.

[21] J. Dukelsky, G. Ortiz, S. M. A. Rombouts, and K. Van Houcke, Phys. Rev. Lett. 96, 180404 (2006). 